\title{
Recent Advances in Combined Photothermal and Photodynamic Therapies against Cancer Using Carbon Nanomaterial Platforms for In Vivo Studies
}

\author{
Lucas D. Dias ${ }^{1, *} \mathbb{C}$, Hilde H. Buzzá ${ }^{1}$, Mirian D. Stringasci ${ }^{1}\left(\mathbb{D}\right.$ and Vanderlei S. Bagnato ${ }^{1,2}$ \\ 1 São Carlos Institute of Physics, University of São Paulo, São Carlos 13566-590, Brazil; \\ hilde.buzza@usp.br (H.H.B.); mirianstringasci@gmail.com (M.D.S.); vander@ifsc.usp.br (V.S.B.) \\ 2 Hagler Fellows, Department of Biomedical Engineering, Texas A\&M University, \\ College Station, TX 77843, USA \\ * Correspondence: lucasdanillodias@gmail.com; Tel.: +1-5516-3373-9823
}

Citation: Dias, L.D.; Buzzá, H.H.; Stringasci, M.D.; Bagnato, V.S. Recent Advances in Combined Photothermal and Photodynamic Therapies against Cancer Using Carbon Nanomaterial Platforms for In Vivo Studies. Photochem 2021, 1, 434-447. https:// doi.org/10.3390/photochem1030026

Academic Editor: Massimo La Deda

Received: 30 August 2021

Accepted: 19 October 2021

Published: 21 October 202

Publisher's Note: MDPI stays neutral with regard to jurisdictional claims in published maps and institutional affiliations.

Copyright: (c) 2021 by the authors. Licensee MDPI, Basel, Switzerland. This article is an open access article distributed under the terms and conditions of the Creative Commons Attribution (CC BY) license (https:// creativecommons.org/licenses/by/ $4.0 /)$.

\begin{abstract}
Cancer is considered one of the major public health problems worldwide. Among the therapeutic approaches investigated and used so far, the combined use of photothermal (PTT) and photodynamic (PDT) therapies have shown promising results for in vivo studies. The mechanisms of actions of both therapies are based on use of a chemical entity and a source light with an appropriate wavelength, and, in PDTs case, also molecular oxygen $\left(\mathrm{O}_{2}\right)$. Moreover, the combined use of PTT and PDT may present a synergic effect on the elimination of solid tumor and metastasis. Herein, we review the past 5 years (2016-2020) regarding the combined use of PTT and PDT and carbon nanomaterial platforms as photosensitizers and photothermal agents against cancer (in vivo evaluation). We intend to highlight the most important and illustrative examples for this period. Additionally, we report the mechanisms of action of PTT and PTT and the general physical/chemical properties of carbon nanomaterial platforms used for this therapeutic approach.
\end{abstract}

Keywords: photothermal therapy; photodynamic therapy; carbon material; cancer; in vivo

\section{Introduction}

Cancer is one of the death-leading diseases, corresponding to about $15 \%$ of total human deaths [1]. The main currently available treatments are surgery, radiotherapy, and chemotherapy. However, these techniques often do not promote complete tumor ablation, in addition to also causing damage to normal cells and several side effects, like infection, inflammation, and drug resistance [2]. Recently, phototherapies such as photodynamic therapy (PDT) and photothermal therapy (PTT) have emerged as promising alternatives and have been recommended in tumor treatment [3-6]. The phototherapies are emerging due to their characteristics such as high specificity, high efficiency, low cost, low toxicity in the dark, remote controllability, no serious side effects, and an absence of contribution to drug resistance [7-9].

The phototherapies can be used together and even in combination with other techniques, achieving greater tumor elimination. These synergistic systems combined can be highly promising due to their enhanced therapeutic efficacy and reduced side effects, because it is possible to obtain better results using lower doses or by the treatment number of individual modes [10-12].

A variety of photosensitizer (PS) and photothermal agents (PA) (e.g., supramolecular polymers, carbon-based materials and porphysomes) [13], as well as both associates, have been investigated to enhance individual and combined therapies [14]. Among them, carbonbased materials have shown promising results when applied in this dual therapy due to their properties such as strong absorption (visible-NIR region), heat and reactive oxygen species (ROS) production, and biocompatibility $[15,16]$. These materials are classified as zero-dimensional (0D) (e.g., fullerene), one-dimensional (1D) (e.g., carbon nanotubes), 
and two-dimensional (2D) (e.g., graphene and its derivatives) [17]. Carbon nanotubes, graphene, fullerene, diamonds, carbon nanohorns and carbon dots are the most commonly applied due to their optical, chemical and biological properties, availability and cost, as observed in our literature search and described in this review.

It is desired that the PS/PA used must simultaneously present great potential in converting light energy into local hyperthermia and generating ROS, to PTT and PDT effects respectively, under visible and near-infrared (NIR) irradiation [18-20], making possible higher selectivity and tumor destruction [12]. Moreover, other incorporations in the drugs have also been explored for smartly delivering to the tumor. These system-todrug releases can be performed under activation of light or by $\mathrm{pH}$ of biological tissue, for example [21].

One of the strategies that have also been adopted is the combination of a third treatment strategy, mainly the chemotherapy $(\mathrm{CT})$, with the phototherapies to obtain a more aggressive treatment $[22,23]$. To find the best way to fully exert the synergetic advantages of each mode it is necessary to optimize the treatment strategy combination. Doxorubicin (DOX) is an anticancer cytotoxic agent widely used in CT, as in the treatment of leukemia, malignant lymphoma, breast, liver, lung, and ovarian cancers [24,25]. However, DOX has poor solubility, strong cytotoxicity to normal human tissue, and nonspecific drug release. Several studies have reported the synthesis of various materials and their conjugation with DOX in the attempt to control and targeted drug release, serving as a targeted drug carrier to reduce unwanted toxicity and enhance specificity to tumor cells [25].

The development of nanostructured materials made it possible to simultaneously diagnose and localize drug delivery in cancer treatment into a single nanoplatform (named nanotheranostics). The development new theranostics to incorporate in the simultaneous PDT / PTT treatment facilitates monitoring the accumulation of the PS and PA in the tumor and guides convenient treatment $[26,27]$. The use of theranostics platforms can make the treatment more specific, more efficient, and reduce the side effects $[28,29]$. One of the most common diagnostic tools that have been used in these platforms is magnetic resonance imaging (MRI), which can screen the tumor in different tissues and organs with great penetration and highly sensitive and accurate images [30]. The presence of theranostic agents such as iron nanoparticles, microbubbles, and radionuclide in the platform favor the precise location of drug with high sensitivity, temporal resolution, target to background ratio and favorable cost-benefit [31].

Currently, the growth and development of nanotechnology gives hope for important achievements in clinical biomedicine with respect to combined use of PTT and PDT. Herein, we review the past 5 years (2016-2020) regarding the combined use of PTT and PDT using carbon nanomaterial platforms such as PS/PA against cancer in vivo studies. Additionally, we report the mechanisms of action of PTT and PDT and general physical/chemical properties of carbon nanomaterial platforms used for this dual therapeutic approach. There are, in the literature, other reviews that describe the combined action of PTT and PDT well [13,32-36], but we believe that a dedicated review comparing the in vivo experiments, including specific parameters such as dose light, PS and PA, with their respective protocols and describing the recent advances in application of carbon-based materials for the combination of PTT and PDT for these in vivo studies is useful and can open new scientific perspectives.

\section{Mechanisms of Photodynamic and Photothermal Therapies}

When light interacts with a molecule, one of the phenomena that can occur is absorption. When the molecule absorbs light, it leaves the lowest-energy state, which is called the ground state $\left(\mathrm{PS}_{0}\right)$, and goes into an excited state with a high energy content (Sn). Within this electronically excited state, there are several sublevels composing the rotational or vibrational states and, by internal conversion phenomenon, coupled with vibrational relaxation, the molecule goes to the lowest excited state (which is called $\mathrm{PS}_{1}$ ) [37]. 
From the molecule in the $S_{1}$ state, three different types of energy dissipation can happen: radiative dissipation, vibration relaxation and intersystem crossing (ISC) [38]. Among these possibilities to return to $S_{0}$, molecules release the absorbed energy in the form of light (radiative process). This process is generically called luminescence, and it can be classified as fluorescence or phosphorescence, depending on spin and energy level settings with consequences on the time of transfer [38]. Fluorescence is when the molecule returns from $S_{1}$ to $S_{0}$ directly by radiative emission and is an optical tool widely used in several types of diagnosis and material characterizations [39,40].

The vibrational relaxation is done by intramolecular movements and collisions with the surrounding molecules, resulting in an increase in kinetic energy and, therefore, in the generation of heat. Thermal effects result from the light energy conversion in heat for a combination of non-radiative processes, such as internal conversion, intersystem crossing and vibrational relaxations, necessarily producing mechanical energy [41]. Then, when thermal energy is generated locally from light irradiation, it is possible to promote thermal damage that ends by leading the cell to death with high selectivity [41]. PTT uses this route and needs a PA to convert light into heat. The development of efficient PA is essential to guaranteeing the effectiveness of treatments and some important PA properties are infrared absorption for great tissue penetration, good heat conversion efficiency, biocompatibility and non-toxicity in the dark [41].

Hyperthermia can also cause indirect cytotoxic effects. Specifically in tumors, the increase in temperature can reduce blood flow and, therefore, lead to hypoxia of tumor cells. These processes make PTT one great new therapeutic option for tumor treatment, when possible, to be done in local matter. Cellular environment temperatures between 42 to $47^{\circ} \mathrm{C}$ is enough to make a large number of cells unfeasible, since high temperatures cause the denaturation of proteins, among other effects [42]. As a selected example, Zhang and co-authors evaluated a gold nanorod as PA against melanoma cells and observed that at $43{ }^{\circ} \mathrm{C}$, the percentages of apoptosis, necroptosis and necrosis of tumor cells were $10.2 \%, 18.3 \%$, and $17.6 \%$, respectively. Whereas, when the temperature increased $49^{\circ} \mathrm{C}$, necrosis was shown as the dominant cell death pathway (52.8\%). Interestingly, when the PTT achieved a moderate temperature of $46^{\circ} \mathrm{C}$, necroptosis was significantly increased (35.1\%) [43]. Moreover, for a PTT protocol, the temperature should be modulated to avoid damage on nearby healthy tissues and intense inflammation response [44].

Another technique that has been increasingly accepted in the treatment of various diseases, especially in the treatment of tumors, is PDT. Its mechanism of action involves the interaction of three elements: light at a specific wavelength, a PS, and the presence of molecular oxygen. This interaction results in photo-physical-chemical reactions that generate reactive oxygen species (ROS) with a high oxidizing power of cellular components, making the cell unviable [37].

For PDT to take place, the molecule goes to its triplet excited state by the so-called intersystem crossing. This event has a low probability according to the selection rules of quantum mechanics and this is a metastable state. From this state it can also return to its ground state by relaxation with the final result in heat or emitting light, which is called phosphorescence. From this metastable state, two types of PDT mechanisms have been proposed to lead to cell death [41]. The type I mechanism describes the generation of oxidative species, namely hydroxyl radical $(\bullet \mathrm{OH})$, hydrogen peroxide $\left(\mathrm{H}_{2} \mathrm{O}_{2}\right)$, and superoxide ion $\left(\mathrm{O}_{2} \bullet-\right)$ via electron transfer [41].

Differently, in the type II mechanism, the excited PS in the metastable triplet state generated by the ISC undergoes quenching (suppression) by transferring energy directly to the triplet oxygen $\left({ }^{3} \mathrm{O}_{2}\right)$, which is in the ground state. Molecular oxygen (in $\mathrm{S}_{0}$ ), with the energy received, goes to a singlet excited state $\left({ }^{1} \mathrm{O}_{2}\right)$, highly cytotoxic and the main mediator of cell damage caused by PDT. In any case, cell death caused by PDT has as its main mechanism of action the production of ${ }^{1} \mathrm{O}_{2}$ and the induction of cell death by apoptosis. These two mechanisms can happen simultaneously and the proportion between 
them is directly related to the oxygen concentration in the cellular environment, the intracellular substrates present, and the characteristics of the PSs chosen in the application.

From the starting absorbed light energy, radiative processes, PDT, and PTT can happen and the combination of these processes can result in therapies combined or in a theranostic agent, with properties of therapy and diagnosis. All of these processes can be conventionally summarized by the Jablonski diagram shown in Figure 1.

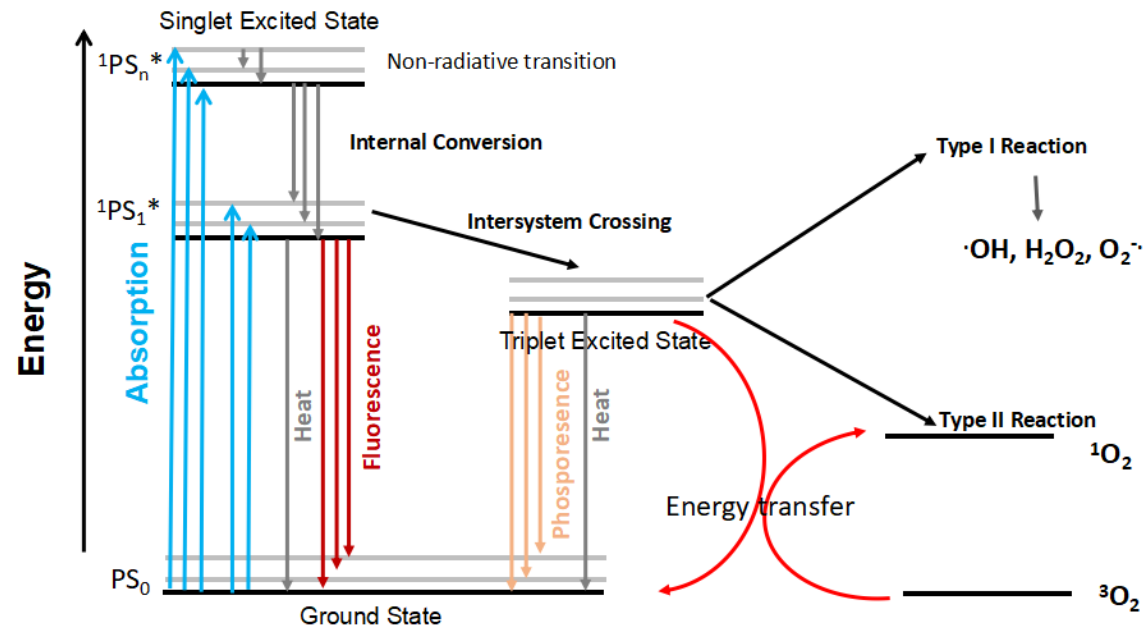

Figure 1. Jablonski Diagram, showing the ways of the molecule from light absorption. Adapted from [45]. ${ }^{1} \mathrm{PSn}{ }^{*}$ : excited state; ${ }^{1} \mathrm{PS}_{1}{ }^{*}$ : lowest excited state; $\mathrm{PS}_{0}$ : ground state.

\section{Carbon-Based Platforms for Photothermal and Photodynamic Therapies}

Carbon-based materials have been mostly evaluated in the Biomedical field (sensing, diagnosis and treatment including PTT and PDT) due to their biocompatibility and physical/chemical properties [46]. When compared to other materials (i.e., supramolecular polymers, porphysomes, cyanine dyes, porphyrins, and melanin), the carbon-based materials present some advantages such as a high cargo loading, high photothermal conversion efficiency, easy modification and functionalization, intrinsic photostability, and structural flexibility $[47,48]$.

There is a wide family of carbon-based materials (allotropes of carbon) such as carbon nanotubes (CNTs), graphene, graphene oxide, diamonds, carbon dots (CQD) and fullerene [49] (Figure 2).

CNTs are hollow cylinders consisting of graphitic sheets and are divided into two main groups (in a structural view): (i) single walled carbon nanotube (SWCNT), which is characterized by a single graphitic sheet showing a high aspect ratio. Moreover, these carbon materials present unique optical, mechanical, electrical and structural diversity, as described in this review paper; (ii) multi walled carbon nanotubes (MWCNT) show multiple layers of graphene, forming a concentric pattern around the smallest, in other words, present multiple SWCNTs nested inside one another [50].

Another example is graphene, which is considered an attractive carbon-based material showing a planar graphitic sheet and a $\mathrm{sp}^{2}$ hybridization carbon network with a carboncarbon distance and interlayer spacing of $1.42 \AA$ and $3.4 \AA$, respectively [50]. In this context, graphene oxide is formed by chemical reactions such as exfoliation and oxidation of layered crystalline graphite [51].

Diamond is a tetrahedral $\mathrm{sp}^{3}$ carbon material, which shows a transparent electrical insulator. Moreover, this material presents on its surface different organic functional groups (e.g., $-\mathrm{COOH},-\mathrm{OH})$, the identity of which depends on the chemical conditions for the purification process [52].

Carbon dots (CQD) possess a mixture of $\mathrm{sp}^{2}$ and $\mathrm{sp}^{3}$ carbon atoms in different ratios [52]. This type of carbon material is formed by zero-dimensional graphene sheets which are characterized by ultra-small sizes and high surface-to-volume ratios [53]. Moreover, car- 
bon dots present a large surface with $\pi$ electrons available which allow immobilization of different chemical entities and biomedical applications such as drug delivery, biomolecules sensing, PTT and PDT [50].

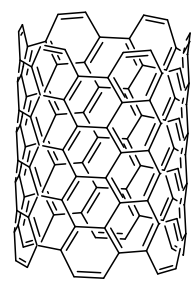

Carbon nanotubes

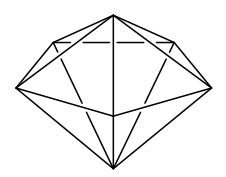

Diamonds

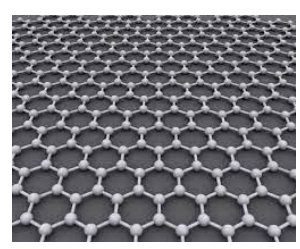

Graphene

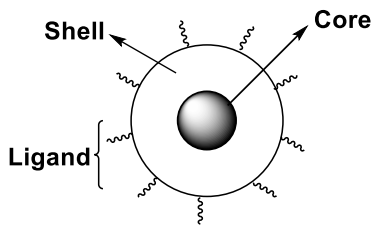

Quantum dots

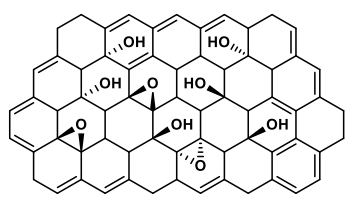

Graphene oxide

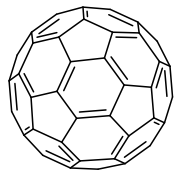

Fullerene

Figure 2. Example of carbon-based materials applied in PDT and PTT [49].

Fullerenes have $\mathrm{sp}^{2}$-hybridized carbon atoms, which are carbon-based materials of pseudospherical symmetry consisting of pentagons and hexagons and are considered the smallest stable carbon-based structures [54]. Moreover, their optical properties are modulated by different factors, e.g., the size and morphology of the nanostructure [52].

All carbon structures are great light absorbers in different regions of the electromagnetic spectrum, which facilitate the use of such materials for many applications. Besides, these carbon-based materials (e.g., graphene) are biocompatible but not approved yet as safe materials for medical applications. Regarding their biodistribution and excretion processes, the carbon-based materials (e.g., carbon nanotubes) when administered intravenously (IV), have a clear accumulation for liver and lung and are excreted via biliary and urinary pathways [55]. On the other hand, using mice as an animal model, Jasim and co-authors showed that graphene derivatives present a spleen accumulation and a urinary excretion $[56,57]$.

\section{In Vivo Studies Using a Combination between Photothermal and Photodynamic Approaches against Cancer}

Due to the wide applicability and efficiency of carbon-based platforms in PTT/PDT, herein, we review the past 5 years (2016-2020) concerning the combined use of PTT and PDT and carbon nanomaterial platforms as PS/PA against cancer (in vivo evaluation). For the purpose of inclusion, we considered the combined use (PTT and PDT) when one of the following criteria were observed: (i) the use of a PS and a PA (carbon material) both irradiated using one wavelength; or (ii) the use of a carbon-based platform as PS and PA being irradiated using two different wavelengths or irradiance. We did not intend to create a comprehensive collection of work; instead, we highlighted the most important and illustrative examples for this period.

Table 1 shows the parameters used (animal model, PS, route of administration, PS/PAlight interval (the time interval between PS/PA administration and light activation) and wavelength/irradiance/time) for combined PTT/PDT protocols using carbon-based platforms such as PS/PA of all papers selected. They are divided into three different sections: the combination of PDT and PTT, the trimode therapy - when PDT and PTT are combined with a third treatment (in this study, only CT was found), and Theranostic-when PDT and PTT are combined with a diagnosis technique. 


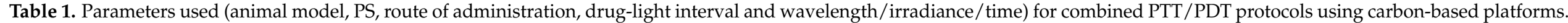

\begin{tabular}{|c|c|c|c|c|c|c|c|c|c|c|c|c|c|}
\hline \multirow[b]{2}{*}{ Entry } & \multirow[b]{2}{*}{$\begin{array}{l}\text { Animal } \\
\text { Lineage }\end{array}$} & \multirow[b]{2}{*}{ Model } & \multicolumn{4}{|c|}{ PDT } & \multicolumn{4}{|c|}{ PTT } & \multirow[b]{2}{*}{$\begin{array}{c}\text { Main } \\
\text { Formulation }\end{array}$} & \multirow[b]{2}{*}{ Used Protocol } & \multirow[b]{2}{*}{ References } \\
\hline & & & $\begin{array}{c}\text { PS/ } \\
\text { Concentration }\end{array}$ & $\begin{array}{l}\text { Route of Ad- } \\
\text { ministration }\end{array}$ & $\begin{array}{c}\text { Drug- } \\
\text { Light } \\
\text { Interval }\end{array}$ & $\begin{array}{l}\text { Wavelenght } \\
\text { (Dose- } \\
\text { Irradiance } \\
\text { and Time) }\end{array}$ & $\begin{array}{c}\text { PS/ } \\
\text { Concentration }\end{array}$ & $\begin{array}{l}\text { Route of Ad- } \\
\text { ministration }\end{array}$ & $\begin{array}{l}\text { PS/PA-Light } \\
\text { Interval }\end{array}$ & $\begin{array}{c}\text { Wavelenght } \\
\text { (Dose- } \\
\text { Irradiance } \\
\text { and Time) }\end{array}$ & & & \\
\hline 1 & $\begin{array}{l}\text { Nude mice } \\
\text { (female: } \\
\text { 6-7 weeks) }\end{array}$ & $\begin{array}{l}\text { 4T1cells into the } \\
\text { hind legs }\end{array}$ & $\begin{array}{c}\text { ICG: } \\
25 \mathrm{ug} / \mathrm{mL}\end{array}$ & $\begin{array}{l}\text { In situ (intra- } \\
\text { tumoral) }\end{array}$ & $\begin{array}{l}4 \mathrm{~h} \text { and } \\
4 \text { days }\end{array}$ & $\begin{array}{c}808 \mathrm{~nm} \\
\left(0.3 \mathrm{~W} / \mathrm{cm}^{2}\right)\end{array}$ & $\begin{array}{l}\text { single-walled } \\
\text { carbon } \\
\text { nanohorns } \\
\text { (SWNH) }\end{array}$ & $\begin{array}{l}\text { In situ (intra- } \\
\text { tumoral) }\end{array}$ & $\begin{array}{l}4 \mathrm{~h} \text { and } \\
4 \text { days }\end{array}$ & $\begin{array}{c}808 \mathrm{~nm} \\
\left(0.3 \mathrm{~W} / \mathrm{cm}^{2}\right)\end{array}$ & PBS & $\begin{array}{l}\text { One irradiation } \\
\text { after } 4 \mathrm{~h} \text { of } \\
\text { injection and a } \\
\text { second irradiation } \\
\text { after } 4 \text { days. }\end{array}$ & [58] \\
\hline 2 & Balb/c mice & $4 \mathrm{~T} 1$ cells & $\begin{array}{l}\text { Chlorin } \\
\text { e6/10 mg/ } \\
\text { kgb.w }\end{array}$ & intravenous & - & $\begin{array}{c}650 \mathrm{~nm} \\
\left(40 \mathrm{~mW} \mathrm{\textrm {cm } ^ { 2 }}\right) \\
\text { for } 10 \mathrm{~min}\end{array}$ & SWNHs & intravenous & - & $\begin{array}{l}808 \mathrm{~nm} \\
\left(0.5 \mathrm{~W} \mathrm{~cm}^{-2}\right. \\
\text { for } 10 \mathrm{~min}) .\end{array}$ & PBS & $\begin{array}{c}\text { PDT + PTT } \\
\text { combination } \\
\text { therapy was } \\
\text { performed by } \\
\text { treating the } \\
\text { tumors with PDT } \\
\text { and PTT } \\
\text { sequentially } \\
\end{array}$ & [59] \\
\hline 3 & $\begin{array}{l}\text { mice (aged } \\
6-7 \text { weeks, } \\
\text { female }\end{array}$ & $4 \mathrm{~T} 1$ cells & $\begin{array}{l}\text { SWNH- } \\
\text { hypericin }\end{array}$ & intratumorally & $\begin{array}{l}\text { Four days } \\
\text { later }\end{array}$ & $\begin{array}{c}590 \mathrm{~nm} \\
\left(0.5 \mathrm{~W} / \mathrm{cm}^{2}\right. \\
\text { for } 5 \mathrm{~min}) .\end{array}$ & $\begin{array}{l}\text { SWNH- } \\
\text { hypericin }\end{array}$ & intratumorally & $\begin{array}{l}\text { Four days } \\
\text { later }\end{array}$ & $\begin{array}{c}808 \mathrm{~nm} \\
\left(0.5 \mathrm{~W} / \mathrm{cm}^{2}\right. \\
\text { for } 5 \mathrm{~min}) .\end{array}$ & saline & $\begin{array}{c}\text { SWNH-Hyp } \\
\text { nanohybrid and } \\
590 / 808 \mathrm{~nm} \\
\text { laser irradiation. }\end{array}$ & [60] \\
\hline 5 & Nude mice & $\begin{array}{l}\text { 4T1 subcutaneous } \\
\text { tumor }\end{array}$ & $\begin{array}{c}\text { chlorin e6/ } \\
2 \mathrm{uM}\end{array}$ & tail vein & $8 \mathrm{~h}$ & $\begin{array}{c}671 \mathrm{~nm} \\
\left(500 \mathrm{~mW} / \mathrm{cm}^{2}\right. \\
\text { for } 10 \mathrm{~min})\end{array}$ & $\begin{array}{c}\text { amino-rich } \\
\text { red emissive } \\
\text { carbon dots } \\
(\mathrm{RCDs}) / \\
2 \mathrm{mg} / \mathrm{mL} \\
\end{array}$ & tail vein & $8 \mathrm{~h}$ & $\begin{array}{c}671 \mathrm{~nm} \\
\left(500 \mathrm{~mW} / \mathrm{cm}^{2}\right. \\
\text { for } 10 \mathrm{~min})\end{array}$ & PBS & $\begin{array}{c}\text { For the } \\
\text { combination, use } \\
\text { of one compound } \\
\text { Ce6-RCDs, with } \\
\text { one irradiation }\end{array}$ & [61] \\
\hline 7 & $\begin{array}{l}\text { Female } \\
\text { BALB/c mice } \\
\text { (8 weeks old, } \\
21-25 \text { g) }\end{array}$ & $\begin{array}{c}\text { 4T1-Luc cells } \\
\left(2 \times 10^{4} \text { cells in }\right. \\
50 \mu \mathrm{L} \text { by } \\
\text { subcutaneous } \\
\text { injection), tumors } \\
\text { treated with } \\
\sim 25 \mathrm{~mm}^{3}\end{array}$ & $\begin{array}{c}\mathrm{MB} \\
(2.5 \mathrm{mg} / \mathrm{kg})\end{array}$ & $\begin{array}{c}\text { Intratumorally } \\
\text { injection }\end{array}$ & $10 \mathrm{~min}$ & $\begin{array}{l}660 \mathrm{~nm} \text { LED } \\
\text { light } \\
\left(90.8 \mathrm{~J} / \mathrm{cm}^{2}\right. \\
\text { for } 10 \mathrm{~min})\end{array}$ & $\begin{array}{l}\text { NanoGO } \\
(10 \mathrm{mg} / \mathrm{kg})\end{array}$ & $\begin{array}{c}\text { Intratumorally } \\
\text { injection }\end{array}$ & $10 \mathrm{~min}$ & $\begin{array}{l}808 \mathrm{~nm} \text { NIR } \\
\text { laser light } \\
\left(8.3 \mathrm{~kJ} / \mathrm{cm}^{2}\right. \\
\text { for } 15 \mathrm{~min})\end{array}$ & $\begin{array}{c}\text { NanoGO-MB } \\
(25 \mu \mathrm{L}, \\
10 \mathrm{mg} / \mathrm{kg} \text { of } \\
\mathrm{NanoGO} \text { and } \\
2.5 \mathrm{mg} / \mathrm{kg} \text { of } \\
\mathrm{MB})\end{array}$ & $\begin{array}{l}\text { PTT was } \\
\text { performed after } \\
\text { the PDT for } \\
\text { combined } \\
\text { PDT/PTT }\end{array}$ & [63] \\
\hline 8 & $\begin{array}{c}\text { Nude } \\
\text { BALB/c mice }\end{array}$ & $\begin{array}{c}\mathrm{KB} \text { cells } \\
\left(2 \times 10^{6} \mathrm{~mL}^{-1}\right) \\
\text { subcutaneously } \\
\text { injected, tumors } \\
\text { treated with } \\
\sim 60 \mathrm{~mm}^{3}\end{array}$ & $\begin{array}{c}\mathrm{Ce} 6 \\
(0.05 \mathrm{mg} / \mathrm{mL})\end{array}$ & $\begin{array}{c}\text { intratumoral } \\
\text { injection }\end{array}$ & $\begin{array}{l}\text { immediately } \\
\text { and } 6 \text { days } \\
\text { after }\end{array}$ & $\begin{array}{l}\text { laser He-Ne } \\
\text { source, } \\
670 \mathrm{~nm}^{2} \\
\left(2 \mathrm{~W} / \mathrm{cm}^{2} \text { for }\right. \\
4 \mathrm{~min}) .\end{array}$ & $\begin{array}{l}\mathrm{ND} / \mathrm{PCM} \\
(5 \mathrm{mg} / \mathrm{kg})\end{array}$ & $\begin{array}{c}\text { intratumoral } \\
\text { injection }\end{array}$ & $\begin{array}{l}\text { immediately } \\
\text { and } 6 \text { days } \\
\text { after }\end{array}$ & $\begin{array}{l}\text { laser He-Ne } \\
\text { source, } \\
670 \mathrm{~nm}^{2} \\
\left(2 \mathrm{~W} / \mathrm{cm}^{2} \text { for }\right. \\
4 \mathrm{~min}) .\end{array}$ & $\begin{array}{c}\mathrm{Ce} 6 / \mathrm{ND} / \mathrm{PCM} \\
(0.1 \mathrm{~mL}, \\
\text { equivalent } \mathrm{ND} \\
5 \mathrm{mg} / \mathrm{kg} \text { body } \\
\text { weight) and } \\
\mathrm{ND} / \mathrm{PCM} \\
(0.1 \mathrm{~mL}, \\
\text { equivalent } \mathrm{ND} \\
5 \mathrm{mg} / \mathrm{kg} \text { body } \\
\text { weight) }\end{array}$ & $\begin{array}{l}\text { The laser was } \\
\text { exposed to tumor } \\
\text { site two times } \\
\text { after post injection } \\
\text { and } 6 \text { days to PDT } \\
\text { e PTT } \\
\text { simultaneous }\end{array}$ & [3] \\
\hline
\end{tabular}


Table 1. Cont.

\begin{tabular}{|c|c|c|c|c|c|c|c|c|c|c|c|c|c|}
\hline \multirow[b]{2}{*}{ Entry } & \multirow[b]{2}{*}{$\begin{array}{l}\text { Animal } \\
\text { Lineage }\end{array}$} & \multirow[b]{2}{*}{ Model } & \multicolumn{4}{|c|}{ PDT } & \multicolumn{4}{|c|}{ PTT } & \multirow[b]{2}{*}{$\begin{array}{c}\text { Main } \\
\text { Formulation }\end{array}$} & \multirow[b]{2}{*}{ Used Protocol } & \multirow[b]{2}{*}{ Reference } \\
\hline & & & $\begin{array}{c}\text { PS/ } \\
\text { Concentration }\end{array}$ & $\begin{array}{l}\text { Route of Ad- } \\
\text { ministration }\end{array}$ & $\begin{array}{c}\text { Drug- } \\
\text { Light } \\
\text { Interval }\end{array}$ & $\begin{array}{l}\text { Wavelenght } \\
\text { (Dose- } \\
\text { Irradiance } \\
\text { and Time) }\end{array}$ & $\begin{array}{c}\text { PS/ } \\
\text { Concentration }\end{array}$ & $\begin{array}{l}\text { Route of Ad- } \\
\text { ministration }\end{array}$ & $\begin{array}{l}\text { PS/PA-Light } \\
\text { Interval }\end{array}$ & $\begin{array}{l}\text { Wavelenght } \\
\text { (Dose- } \\
\text { Irradiance } \\
\text { and Time) }\end{array}$ & & & \\
\hline 9 & nude mice & $\begin{array}{l}\text { HeLa tumor, } \\
\text { tumor treated } \\
\text { with } 20-25 \mathrm{~mm}^{3}\end{array}$ & $\begin{array}{l}\mathrm{CQD}(2 \\
\mathrm{mg} / \mathrm{mL}, \\
100 \mu \mathrm{L})\end{array}$ & $\begin{array}{l}\text { intravenous } \\
\text { injection via } \\
\text { the tail vein }\end{array}$ & $6 \mathrm{~h}$ & $\begin{array}{l}635 \mathrm{~nm} \text { laser } \\
\left(0.1 \mathrm{~W} / \mathrm{cm}^{2}\right. \\
\text { for } 10 \mathrm{~min})\end{array}$ & $\begin{array}{l}\mathrm{CQD}(2 \\
\mathrm{mg} / \mathrm{mL}, \\
100 \mu \mathrm{L})\end{array}$ & $\begin{array}{l}\text { intravenous } \\
\text { injection via } \\
\text { the tail vein }\end{array}$ & $6 \mathrm{~h}$ & $\begin{array}{l}635 \mathrm{~nm} \text { laser } \\
\left(2 \mathrm{~W} / \mathrm{cm}^{2} \text { for }\right. \\
10 \mathrm{~min})\end{array}$ & $\begin{array}{l}\mathrm{CQD}(2 \\
\mathrm{mg} / \mathrm{mL} \\
100 \mu \mathrm{L})\end{array}$ & $\begin{array}{l}635 \mathrm{~nm} \text { laser } \\
\left(2 \mathrm{~W} / \mathrm{cm}^{2} \text { for }\right. \\
10 \mathrm{~min}) \text { by } \\
\text { PDT/PTT } \\
\text { simultaneous } \\
\end{array}$ & [64] \\
\hline 10 & Nude mice & $\begin{array}{l}\text { subcutaneously } \\
\text { injecting HeLa } \\
\text { cells }\end{array}$ & Free Ce6 & intrevenous & $24 \mathrm{~h}$ & $\begin{array}{l}660 \mathrm{~nm}, \\
\left(1 \mathrm{~W} / \mathrm{cm}^{2} \text { for }\right. \\
10 \mathrm{~min})\end{array}$ & $\begin{array}{l}\text { Carbon } \\
\text { nanotubes } \\
\text { (CNTs), }\end{array}$ & intravenous & $24 \mathrm{~h}$ & $\begin{array}{c}808 \mathrm{~nm} \\
\left(1.0 \mathrm{~W} / \mathrm{cm}^{2}\right. \\
\text { for } 10 \mathrm{~min})\end{array}$ & PBS & $\begin{array}{l}\text { Each treatment } \\
\text { was made } \\
\text { separetely and the } \\
\text { combination was } \\
\text { made with a } \\
\text { molecue } \\
\text { Ce6-MnO2/ } \\
\text { CNTs (CMCs) } \\
\text { following the } \\
\text { ioradiation with } \\
\text { both wavelenght }\end{array}$ & [65] \\
\hline 11 & $\begin{array}{l}\text { female nude } \\
\text { mice }\end{array}$ & $\begin{array}{l}1 \times 10^{6} \text { human } \\
\text { liver cancer cells } \\
\text { (HeLa) implanted } \\
\text { subcutaneously in } \\
\text { the left and right } \\
\text { axillaries of nude } \\
\text { mice. Tumors } \\
\text { treated with } \\
100-120 \mathrm{~mm}^{3} \\
\end{array}$ & $\mathrm{Rf}$ & $\begin{array}{l}\text { tail vein } \\
\text { injection }\end{array}$ & $8 \mathrm{~h}$ & $\begin{array}{c}671 \mathrm{~nm} \text { laser } \\
\left(2 \mathrm{~W} / \mathrm{cm}^{2} \text { for }\right. \\
8 \mathrm{~min})\end{array}$ & FeN@CQD & $\begin{array}{l}\text { tail vein } \\
\text { injection }\end{array}$ & $8 \mathrm{~h}$ & $\begin{array}{l}671 \mathrm{~nm} \text { laser } \\
\left(2 \mathrm{~W} / \mathrm{cm}^{2} \text { for }\right. \\
8 \mathrm{~min})\end{array}$ & $\begin{array}{c}\text { GP-Rf-FA- } \\
\text { FeN@CQDs } \\
\text { (1 mg/mL) and } \\
\text { GP-Rf-FA- } \\
\text { FeN@CQDs- } \\
\text { DOX } \\
(1 \mathrm{mg} / \mathrm{mL})\end{array}$ & $\begin{array}{l}\text { PDT and PTT } \\
\text { simultaneous }\end{array}$ & [66] \\
\hline 12 & Nude mice & Hela cells & $\begin{array}{c}\text { SWCNTs- } \\
\text { PEG- } \\
\text { Fe3O4@CQDs: } \\
100 \mu \mathrm{g} / \mathrm{mL} \\
100 \mu \mathrm{L}\end{array}$ & $\begin{array}{l}\text { Intravenous } \\
\text { via tail vein }\end{array}$ & - & $\begin{array}{c}808 \mathrm{~nm} \\
\left(0.5 \mathrm{~W} / \mathrm{cm}^{2}\right. \\
\text { for } 5 \mathrm{~min})\end{array}$ & $\begin{array}{c}\text { SWCNTs- } \\
\text { PEGFe3O4@ } \\
\text { CQDS/DOX- } \\
\text { Apt: } \\
100 \mu \mathrm{g} / \mathrm{mL} \\
100 \mu \mathrm{L}\end{array}$ & $\begin{array}{l}\text { Intravenous } \\
\text { via tail vein }\end{array}$ & - & $\begin{array}{l}808 \mathrm{~nm} \\
\left(2 \mathrm{~W}^{2} / \mathrm{cm}^{2} \text { for }\right. \\
5 \mathrm{~min})\end{array}$ & PBS & $\begin{array}{l}\text { They treated with } \\
\text { only PDT, only CT, } \\
\text { PDT and CT and } \\
\text { irradiation with } \\
\text { both laser power } \\
\left(0.5 \mathrm{~W} / \mathrm{cm}^{2} \text { and }\right. \\
\left.2 \mathrm{~W} / \mathrm{cm}^{2}\right) \text { for } \\
\text { trimode therapy. }\end{array}$ & [67] \\
\hline 13 & $\begin{array}{l}\text { Female } \\
\text { Kunming } \\
\text { mice }\end{array}$ & $\begin{array}{l}\text { subcutaneously } \\
\text { injecting U14 cells } \\
\text { in the left axilla, } \\
\text { tumor treated } \\
\text { with diameter } \\
\sim 6-10 \mathrm{~mm}\end{array}$ & $\begin{array}{c}\text { GSC } \\
(0.1 \mathrm{~mL} \\
1000 \mu \mathrm{g} / \mathrm{mL})\end{array}$ & $\begin{array}{l}\text { intravenous } \\
\text { injection }\end{array}$ & $1 \mathrm{~h}$ & $\begin{array}{l}650 \mathrm{~nm} \text { laser } \\
\text { irradiation } \\
\left(0.5 \mathrm{~W} / \mathrm{cm}^{2}\right. \\
\text { for } 10 \mathrm{~min})\end{array}$ & $\begin{array}{l}\text { DOX-GSCCP } \\
(0.1 \mathrm{~mL} \\
1000 \mu \mathrm{g} / \mathrm{mL})\end{array}$ & $\begin{array}{l}\text { intravenous } \\
\text { injection }\end{array}$ & $1 \mathrm{~h}$ & $\begin{array}{l}980 \mathrm{~nm} \text { laser } \\
\text { irradiation } \\
\left(0.5 \mathrm{~W} / \mathrm{cm}^{2}\right. \\
\text { for } 10 \mathrm{~min})\end{array}$ & $\begin{array}{l}\text { DOX-GSCCP } \\
(0.1 \mathrm{~mL}, \\
1000 \mathrm{\mu g} / \mathrm{mL})\end{array}$ & $\begin{array}{l}650 \text { and } 980 \mathrm{~nm} \\
\text { laser irradiation }\end{array}$ & [24] \\
\hline
\end{tabular}


Table 1. Cont

\begin{tabular}{|c|c|c|c|c|c|c|c|c|c|c|c|c|c|}
\hline \multirow[b]{2}{*}{ Entry } & \multirow[b]{2}{*}{$\begin{array}{l}\text { Animal } \\
\text { Lineage }\end{array}$} & \multirow[b]{2}{*}{ Model } & \multicolumn{4}{|c|}{ PDT } & \multicolumn{4}{|c|}{ PTT } & \multirow[b]{2}{*}{$\begin{array}{c}\text { Main } \\
\text { Formulation }\end{array}$} & \multirow[b]{2}{*}{ Used Protocol } & \multirow[b]{2}{*}{ Reference } \\
\hline & & & $\begin{array}{c}\text { PS/ } \\
\text { Concentration }\end{array}$ & $\begin{array}{l}\text { Route of Ad- } \\
\text { ministration }\end{array}$ & $\begin{array}{c}\text { Drug- } \\
\text { Light } \\
\text { Interval }\end{array}$ & $\begin{array}{l}\text { Wavelenght } \\
\text { (Dose-- } \\
\text { Irradiance } \\
\text { and Time) }\end{array}$ & $\begin{array}{c}\text { PS/ } \\
\text { Concentration }\end{array}$ & $\begin{array}{l}\text { Route of Ad- } \\
\text { ministration }\end{array}$ & $\begin{array}{l}\text { PS/PA-Light } \\
\text { Interval }\end{array}$ & $\begin{array}{l}\text { Wavelenght } \\
\text { (Dose- } \\
\text { Irradiance } \\
\text { and Time) } \\
\end{array}$ & & & \\
\hline 14 & $\begin{array}{l}\text { Balb/c nude } \\
\text { mice }\end{array}$ & HeLa cells & $\begin{array}{l}\text { Chlorin e6/ } \\
5 \mathrm{mg} / \mathrm{kg}\end{array}$ & intravenous & $\begin{array}{l}0,4 \text { and } \\
8 \text { days }\end{array}$ & $\begin{array}{c}660 \mathrm{~nm} \\
\left(100 \mathrm{~mW} / \mathrm{cm}^{2}\right) \\
\text { for } 5 \mathrm{~min}\end{array}$ & $\begin{array}{c}\text { Carbon } \\
\text { nanotubes }\end{array}$ & intravenous & $\begin{array}{l}0,4 \text { and } \\
8 \text { days }\end{array}$ & $\begin{array}{c}808 \mathrm{~nm}^{-2} \\
(1 \mathrm{~W} \mathrm{~cm}) \\
\text { for } 5 \mathrm{~min}\end{array}$ & Saline & $\begin{array}{c}\text { The tumors were } \\
\text { irradiated with } \\
660 \mathrm{~nm} \\
\left(100 \mathrm{~mW} \cdot \mathrm{cm}^{-2}\right) \\
\text { and } / \mathrm{or}^{-108} \mathrm{~nm} \\
\left(1 \mathrm{~W} \cdot \mathrm{cm}^{-2}\right) \mathrm{NIR} \\
\text { laser for } 5 \mathrm{~min} \\
\text { each } 24 \mathrm{~h} \text { after } \\
\text { every injection }\end{array}$ & [68] \\
\hline 15 & $\begin{array}{l}\text { male } \\
\text { C57BL/6 } \\
\text { mice }\end{array}$ & $\begin{array}{c}\text { subcutaneous } \\
\text { HeLa tumor by } \\
\text { injecting of } 1 \times 10^{6} \\
\text { HeLa cells } \\
(200 \mu \mathrm{LL}) \text {, treated } \\
\text { with } 100 \mathrm{~mm}^{3}\end{array}$ & $\begin{array}{c}\text { GP-PGA- } \\
\text { Fe3O4- } \\
\text { CDs@BPQDs } \\
(50 \mu \mathrm{g} / \mathrm{mL})\end{array}$ & $\begin{array}{c}\text { intratumoral } \\
\text { injection }\end{array}$ & $2.5 \mathrm{~h}$ & $\begin{array}{c}\text { laser- } \\
\text { irradiated at } \\
660 \mathrm{~nm} \\
\left(0.5 \mathrm{~W} / \mathrm{cm}^{2}\right. \\
\text { for } 15 \mathrm{~min})\end{array}$ & $\begin{array}{l}\text { GP-PGA- } \\
\text { Fe3O4- } \\
\text { CDs@BPQDs } \\
(50 \mu \mathrm{g} / \mathrm{mL})\end{array}$ & $\begin{array}{l}\text { intratumoral } \\
\text { injection }\end{array}$ & $2 \mathrm{~h}$ & $\begin{array}{c}\text { laser- } \\
\text { irradiated at } \\
808 \mathrm{~nm} \\
\left(1 \mathrm{~W} / \mathrm{cm}^{2} \text { for }\right. \\
5 \mathrm{~min})\end{array}$ & $\begin{array}{l}\text { GP-PGA-Fe3O4- } \\
\text { CDs@BPQDs } \\
(50 \mu \mathrm{g} / \mathrm{mL})\end{array}$ & $\begin{array}{c}\text { PTT was } \\
\text { permormed } 2 \mathrm{~h} \\
\text { after the injection } \\
\text { while PDT was } \\
\text { performed } 2.5 \mathrm{~h}\end{array}$ & [10] \\
\hline
\end{tabular}




\subsection{Photothermal and Photodynamic Therapies}

Different types of carbon nanomaterials, such as single-walled carbon nanohorns (SWNH), have been used in the construction of nanoplatforms for different types of phototherapies, especially when they present high photothermal conversion efficiency. Using these platform to make indocyanine green (ICG) more stable, Gao and co-authors (Table 1, entry 1) demonstrated superior advantages of the use of SWNH-ICG for synergistic antitumor effects using PTT and PDT in breast tumor cells using low laser power. As described in the literature, the SWNH-ICG nanohybrid materials could generate hyperthermia effects and abundant ROS generation under light source irradiation. The use of a nanohybrid could produce a stronger temperature response compared to free ICG at a low power density. The treatment induced the killing and the growth inhibition of the tumor [58].

In 2020, Yang and co-authors reported the immobilization of Chlorine (Ce6) and Gd $\mathrm{d}^{3+}$ onto polymer-coated SWNHs and evaluated the combination of PDT and PTT against tumors (Table 1, entry 2), in addition to the effect on the immunological system after use of this combined therapy. The authors observed that this synthetized material produces a strong immune adjuvant action and presents a high tumor targeting and penetration efficiency [59].

The synthesis of a hybrid material based on the immobilization of hypericin onto SWNH (Table 1, entry 3) was reported, in 2019, by Gao and co-authors. This material was applied as a dual agent (PDT and PTT) using a light irradiation at 590 and $808 \mathrm{~nm}$ against 4T1 cells injected subcutaneously in mice as tumor models. The authors observed that hypericin immobilized onto SWNH could produce a simultaneous effect (hyperthermia and ROS production) against cancer and the best result was obtained when both wavelengths were used (590 and $808 \mathrm{~nm}$ ) [60].

Sun and co-authors combined Ce6 and CQD to reduce the irradiation of PTT combined with PDT (Table 1, entry 5). While the treatment with PDT or PTT separately was not enough for the tumor reduction, it was clear that the synergic result of treatments were combined, reducing 30\% of the initial volume. These results demonstrated that the efficacy of the developed hybrid material for the synergistic PDT/PTT protocol is better than a single therapeutic protocol (PDT or PTT) under a light source irradiation [61].

The irradiation of GO-PEG-folate can generate singlet oxygen or heat (or both), depending on the excitation wavelengths used ( $808 \mathrm{~nm}$ or $980 \mathrm{~nm}$ ), according to Kalluru and co-authors (Table 1, entry 6). In the treatment of melanoma murine, the combination of PDT/PTT was demonstrated to be more effective, leaving only 0.02 of the size of the initial tumor, if compared to PTT alone (via $808 \mathrm{~nm}$ light irradiation), which reached 10.1-fold the initial size [62].

Some studies have shown that nanographene oxide (NanoGO) immobilized with different PSs are effective in PDT/PTT treatment. Dos Santos and co-authors (Table 1, entry 7) used methylene blue (MB) in association with NanoGO (NanoGO-MB) as a carbon based platform in a murine mammary carcinoma model, which mimics tumor growth and metastasis of stage IV human breast cancer. The combined application of PDT/PTT therapies using NanoGO-MB as a dual agent and promoted complete tumor ablation in $5 / 5$ animals without regrowth and metastasis, suggesting a potential clinical application against breast cancer [63].

Ruy and co-authors (Table 1, entry 8) performed a study using phase-change material (PCM) nanoparticles containing Ce6 and nanodiamonds (NDs) for photodynamic and photothermal effects. Ce6/ND/PCM was demonstrated to be promising due to the synergistic effect of PDT/PTT. After 12 days of treatment, the tumors treated with ND/PCM presented a volume of $50.1 \pm 9.3 \mathrm{~mm}^{3}$, while the tumors treated with Ce6/ND/PCM had a volume of $10.5 \pm 3.5 \mathrm{~mm}^{3}$, suggesting the synergistic effect of PDT/PTT and confirming a superior therapeutic efficiency if compared to only PTT [3].

CQD was obtained from polythiophene benzoic acid (PBA) by Ge and co-authors (Table 1, entry 9). CQD materials simultaneously presented photodynamic and photothermal effects under $635 \mathrm{~nm}$ irradiation, changing only the irradiation $\left(0.1\right.$ or $\left.2 \mathrm{~W} / \mathrm{cm}^{2}\right)$. It 
verified the efficiency of $27 \%$ in ${ }^{1} \mathrm{O}_{2}$ generation and $36.2 \%$ in photothermal conversion. In the PDT/PTT group, it was observed that all tumor cells were damaged. In the PDT group, only some inflammatory cell infiltrations, cell death, and cirrhosis with tissue structure damage could be observed [64].

In the same way, using $\mathrm{MnO}_{2}$ to avoid hypoxia of the tumor, a compound with Ce6 and CNTs was synthesized to analyze the synergism between PDT and PTT (Table 1, entry 10). Yin and co-authors showed the inhibition of the tumor growth with each therapy separated and the best result with a compound combined a PS, a PA and $\mathrm{MnO}_{2}$, Ce6$\mathrm{MnO}_{2} / \mathrm{CNTs}$ (CMCs), following two irradiations (660 and $808 \mathrm{~nm}$ to activate both of therapies). The treated group showed an inhibition in the tumor growth for all irradiated groups, but the use of CMC presented the best result, with a synergic effect between PDT and PTT [65].

Therefore, in all related papers, the combination of PDT and PTT improved the tumor reduction rate in animal models. To improve this effect, these phototherapies can be used with another therapy, such as chemotherapy.

\subsection{Trimode Treatment}

Some studies in the last few years have been associating the third technique with combined PDT/PTT, especially CT, to obtain an even more efficient treatment. This combination (PDT/PTT/CT) can be the possibility to reduce the laser power and the drug dosage. Zhang and co-authors (Table 1, entry 11) prepared fluorescent FeN@CQDs by a green methodology using iron crosslinked chitosan (Ch-Fe-CL). FeN@CQDs was functionalized with folic acid (FA), a targeting ligand, conjugated with riboflavin (Rf) as PS, and was effectively loaded to DOX. The GP-Rf-FA-FeN@CQDs-DOX was developed to realize the PDT/PTT/CT combination and demonstrated a strong NIR absorption and a great photothermal effect. For in vivo study, GP-Rf-FA-FeN@CQDs-DOX demonstrated the ability to eradicate tumors without regrowth and achieved a synergistic therapeutic effect, promising results for clinical applications [66].

Zhang and co-authors (Table 1, entry 12) showed a combination of PDT, PTT and CT effects. SWCNTs and carbon quantum dots (CQDs) have been used as promising nanoplatforms to combine therapies. SWCNTs-PEGFe3O4@CQDs-DOX-Apt was synthesized to provide good selectivity in tumor cells and water-solubility, magnetic properties for MR imaging and the combination of all agents (CNTs, QD and DOX). Although all irradiated groups showed better results than only CT treatment, the trimode combination reached a total elimination of the tumor, showing this to be the best option for tumor treatment [67].

Yang and co-authors (Table 1, entry 13) also performed a new nanoplatform with a multimode PDT/PTT/CT effect composed of anticancer drug DOX, Ce6 as PS, and $\mathrm{CQD}$ as a PA. The platform was constructed by $\mathrm{SiO}_{2}$-Gd-Ce6-CD-P yolk-shell hybrid spheres to control the release of the antidrug at a normal body temperature $\left(37^{\circ} \mathrm{C}\right)$ and not present hemolysis activity to human red blood cells. Moreover, the presence of $\mathrm{Gd}^{3+}$ in the composite generated signals to MRI and X-ray-computed tomography to guided therapy. In an application in murine tumors, it was observed that the size of the tumors treated with PDT/PTT/CT was smaller than those treated with PDT alone or PTT/CT. The results provided suggest a potential platform for future clinical therapy [24].

\subsection{Theranostic: PDT, PTT and Diagnosis Methods}

In addition to performing an effective treatment, there is an interest in having efficient ways to precisely locate the tumor and to track the nanoparticles in the body. Thus, ways to detect nanoparticles through images have been built, and the main techniques that have been used are fluorescence and MR images. For this, nanomaterials/nanoparticles are built that generate these signals.

Wang and co-authors reported the synthesis of a multifunctional nanomaterial (CNT@MnO ${ }_{2}$-PEG@Ce6) for PDT, PTT and MRI. This nanoplatform possesses carbon nanotubes, a $\mathrm{MnO}_{2}$ layer, PEG and Ce6 as PS. Ce6 and $\mathrm{Mn}^{2+}$ are rapidly released due to 
their low $\mathrm{pH}$ and high concentration of glutathione (present in tumor environment) for PDT and MRI, respectively. The authors applied this multifunctional material to evaluate the combined PDT and PTT effects using the protocol conditions described in Table 1, entry 14. According to the authors, the combined use of CNT@MnO $2-\mathrm{PEG@Ce6} \mathrm{and} \mathrm{two}$ wavelength sources (at 660 and $808 \mathrm{~nm}$ ) led to a drastic reduction in tumor growth rate through a synergistic PDT/PTT action [68].

Zhang and co-authors (Table 1, entry 15) used a highly dispersed suspension of ultrasmall black phosphorus quantum dots (BPQDs) that have the unique property of absorbing NIR light with a high tissue-penetration depth. The selective localization of $\mathrm{BPQD}$ could be observed by fluorescence and MR images. The nanoplatform GP-PGA$\mathrm{Fe}_{3} \mathrm{O}_{4}$-CDs@BPQDs presented an efficient generation of ${ }^{1} \mathrm{O}_{2}$ under $660 \mathrm{~nm}$ irradiation and a high PT conversion under $808 \mathrm{~nm}$ irradiation. In a study using murine tumor models, this nanoplatform exhibited a great therapeutic effect and selectivity when compared to commercial drugs, benefiting from the synergistic combination of PDT/PTT. In addition, GP-PGA- $\mathrm{Fe}_{3} \mathrm{O}_{4}$-CDs@BPQDs did not show long-term inflammatory responses or obvious damage [10].

\section{Conclusions and Future Perspectives}

In this literature survey and explanation of the combined use of therapies that start from the principle of converting light energy into energy to cause tumor destruction by hyperthermia and photodynamic action, using carbon-based structures such as PA in combination with several PSs, has been shown to be quite promising and generally advantageous. These carbon-based materials can be co-administered with chemotherapy drugs (e.g., doxorubicin) for a synergistic protocol of PDT/PTT/chemotherapy to increase the anticancer effect. It seems that the combination of PTT with PDT in various forms could bring an improvement in the final result, which is certainly worth further exploration and translation into clinical test models. The various platforms that combine carbon nanostructures with light and PDT may come to compose a varied list of possibilities that should include the diagnosis along with the treatment.

Among them, the toxicity tolerances of these structures is a concern that should be considered in future studies. Furthermore, it is necessary to investigate the independence of the clearance of photosensitizers in the presence of nanostructures together. In this review paper, we have described the combined use of PTT and PTT results in a synergic therapeutic effect for combating cancer cells in vivo studies.

Author Contributions: Conceptualization, L.D.D., H.H.B., M.D.S., V.S.B.; investigation, L.D.D., H.H.B., M.D.S., V.S.B.; resources, L.D.D., H.H.B., M.D.S., V.S.B.; data curation, L.D.D., H.H.B., M.D.S., V.S.B.; writing—original draft preparation, L.D.D., H.H.B., M.D.S., V.S.B.; writing-review and editing, L.D.D., H.H.B., M.D.S., V.S.B.; visualization, L.D.D., H.H.B., M.D.S., V.S.B.; supervision, L.D.D., H.H.B., M.D.S., V.S.B.; project administration, L.D.D.; funding acquisition, L.D.D., H.H.B., M.D.S., V.S.B. All authors have read and agreed to the published version of the manuscript.

Funding: This research was funded by the Brazilian agencies (scholarships and facility infrastructure) via the grants Center for Research in Optics and Photonics-CePOF (Sao Paulo Research FoundationFAPESP grant 2013/07276-1), National Institute of Optics and Photonics-INCT (National Council for Research and Development-CNPq grant 465360/2014-9 and São Paulo Research FoundationFAPESP grants 2014/50857-8, 2016/14033-6 and 2019/13569-8 L.D.Dias thanks to Fundação de Amparo à Pesquisa do Estado de São Paulo (FAPESP) for Post-doctoral grant 2019/13569-8.

Institutional Review Board Statement: Not applicable.

Informed Consent Statement: Not applicable.

Conflicts of Interest: The authors declare no conflict of interest. The funders had no role in the design of the study; in the collection, analyses, or interpretation of data; in the writing of the manuscript, or in the decision to publish the results. 


\section{References}

1. Fitzmaurice, C.; Abate, D.; Abbasi, N.; Abbastabar, H.; Abd-Allah, F.; Abdel-Rahman, O.; Abdelalim, A.; Abdoli, A.; Abdollahpour, I.; Abdulle, A.S.M.; et al. Global, Regional, and National Cancer Incidence, Mortality, Years of Life Lost, Years Lived With Disability, and Disability-Adjusted Life-Years for 29 Cancer Groups, 1990 to 2017 A Systematic Analysis for the Global Burden of Disease Study. JAMA Oncol. 2019, 5, 1749-1768. [PubMed]

2. Lv, Y.; Tao, L.; Bligh, S.W.A.; Yang, H.H.; Pan, Q.X.; Zhu, L.M. Targeted delivery and controlled release of doxorubicin into cancer cells using a multifunctional graphene oxide. Mater. Sci. Eng. C-Mater. Biol. Appl. 2016, 59, 652-660. [CrossRef] [PubMed]

3. Ryu, T.K.; Baek, S.W.; Kang, R.H.; Jeong, K.Y.; Jun, D.R.; Choi, S.W. Photodynamic and photothermal tumor therapy using phase-change material nanoparticles containing chlorin e6 and nanodiamonds. J. Control. Release 2018, 270, 237-245. [CrossRef]

4. Shi, J.J.; Kantoff, P.W.; Wooster, R.; Farokhzad, O.C. Cancer nanomedicine: Progress, challenges and opportunities. Nat. Rev. Cancer 2017, 17, 20-37. [CrossRef] [PubMed]

5. Guo, W.; Guo, C.S.; Zheng, N.N.; Sun, T.D.; Liu, S.Q. CsxWO3 Nanorods Coated with Polyelectrolyte Multilayers as a Multifunctional Nanomaterial for Bimodal ImagingGuided Photothermal/Photodynamic Cancer Treatment. Adv. Mater. 2017, 29 , 1604157. [CrossRef]

6. Romero, M.P.; Buzza, H.H.; Stringasci, M.D.; Estevao, B.M.; Silva, C.C.C.; Pereira-da-Silva, M.A.; Inada, N.M.; Bagnato, V.S. Graphene Oxide Theranostic Effect: Conjugation of Photothermal and Photodynamic Therapies Based on an in vivo Demonstration. Int. J. Nanomed. 2021, 16, 1601-1616. [CrossRef]

7. Dolmans, D.; Fukumura, D.; Jain, R.K. Photodynamic therapy for cancer. Nat. Rev. Cancer 2003, 3, 380-387. [CrossRef]

8. Sharman, W.M.; Allen, C.M.; van Lier, J.E. Photodynamic therapeutics: Basic principles and clinical applications. Drug Discov. Today 1999, 4, 507-517. [CrossRef]

9. Cheng, L.; Wang, C.; Feng, L.Z.; Yang, K.; Liu, Z. Functional Nanomaterials for Phototherapies of Cancer. Chem. Rev. 2014, 114, 10869-10939. [CrossRef]

10. Zhang, M.; Wang, W.T.; Cui, Y.J.; Zhou, N.L.; Shen, J. Near-infrared light-mediated photodynamic/photothermal therapy nanoplatform by the assembly of $\mathrm{Fe}_{3} \mathrm{O}_{4}$ carbon dots with graphitic black phosphorus quantum dots. Int. J. Nanomed. 2018, 13, 2803-2819. [CrossRef]

11. Liu, Y.L.; Zhi, X.; Yang, M.; Zhang, J.P.; Lin, L.N.; Zhao, X.; Hou, W.X.; Zhang, C.L.; Zhang, Q.; Pan, F.; et al. Tumor-triggered drug release from calcium carbonate-encapsulated gold nanostars for near-infrared photodynamic/photothermal combination antitumor therapy. Theranostics 2017, 7, 1650-1662. [CrossRef] [PubMed]

12. Yan, F.; Wu, H.; Liu, H.M.; Deng, Z.T.; Liu, H.; Duan, W.L.; Liu, X.; Zheng, H.R. Molecular imaging-guided photother$\mathrm{mal} /$ photodynamic therapy against tumor by iRGD-modified indocyanine green nanoparticles. J. Control. Release 2016, 224, 217-228. [CrossRef] [PubMed]

13. Yang, Z.Z.; Sun, Z.R.; Ren, Y.; Chen, X.; Zhang, W.; Zhu, X.H.; Mao, Z.W.; Shen, J.L.; Nie, S.N. Advances in nanomaterials for use in photothermal and photodynamic therapeutics. Mol. Med. Rep. 2019, 20, 5-15. [CrossRef]

14. Zhu, W.; Liu, Y.; Yang, Z.; Zhang, L.; Xiao, L.J.; Liu, P.; Wang, J.; Yi, C.F.; Xu, Z.S.; Ren, J.H. Albumin/sulfonamide stabilized iron porphyrin metal organic framework nanocomposites: Targeting tumor hypoxia by carbonic anhydrase IX inhibition and T-1-T-2 dual mode MRI guided photodynamic/photothermal therapy. J. Mater. Chem. B 2018, 6, 265-276. [CrossRef] [PubMed]

15. Liu, Z.; Robinson, J.T.; Tabakman, S.M.; Yang, K.; Dai, H.J. Carbon materials for drug delivery \& cancer therapy. Mater. Today 2011, 14, 316-323.

16. Rajakumar, G.; Zhang, X.H.; Gomathi, T.; Wang, S.F.; Ansari, M.A.; Mydhili, G.; Nirmala, G.; Alzohairy, M.A.; Chung, I.M. Current Use of Carbon-Based Materials for Biomedical Applications-A Prospective and Review. Processes 2020, 8, 355. [CrossRef]

17. Kumar, S.; Saeed, G.; Zhu, L.; Hui, K.N.; Kim, N.H.; Lee, J.H. OD to 3D carbon-based networks combined with pseudocapacitive electrode material for high energy density supercapacitor: A review. Chem. Eng. J. 2021, 403, 126352. [CrossRef]

18. Master, A.; Livingston, M.; Sen Gupta, A. Photodynamic nanomedicine in the treatment of solid tumors: Perspectives and challenges. J. Control. Release 2013, 168, 88-102. [CrossRef]

19. Jang, B.; Park, J.Y.; Tung, C.H.; Kim, I.H.; Choi, Y. Gold Nanorod-Photosensitizer Complex for Near-Infrared Fluorescence Imaging and Photodynamic/Photothermal Therapy In Vivo. Acs Nano 2011, 5, 1086-1094. [CrossRef]

20. Chen, Q.; Wang, C.; Cheng, L.; He, W.W.; Cheng, Z.; Liu, Z. Protein modified upconversion nanoparticles for imaging-guided combined photothermal and photodynamic therapy. Biomaterials 2014, 35, 2915-2923. [CrossRef]

21. Liu, X.D.; Yang, G.B.; Zhang, L.F.; Liu, Z.; Cheng, Z.P.; Zhu, X.L. Photosensitizer cross-linked nano-micelle platform for multimodal imaging guided synergistic photothermal/photodynamic therapy. Nanoscale 2016, 8, 15323-15339. [CrossRef]

22. Yang, J.M.; Yang, H.; Lin, L.W. Quantum Dot Nano Thermometers Reveal Heterogeneous Local Thermogenesis in Living Cells. Acs Nano 2011, 5, 5067-5071. [CrossRef] [PubMed]

23. Jin, C.S.; Lovell, J.F.; Chen, J.; Zheng, G. Ablation of Hypoxic Tumors with Dose-Equivalent Photothermal, but Not Photodynamic, Therapy Using a Nanostructured Porphyrin Assembly. ACS Nano 2013, 7, 2541-2550. [CrossRef] [PubMed]

24. Yang, D.; Yang, G.X.; Gai, S.L.; He, F.; Lv, R.C.; Dai, Y.L.; Yang, P.P. Imaging-Guided and Light-Triggered Chemo/Photodynamic/Photothermal Therapy Based on Gd (III) Chelated Mesoporous Silica Hybrid Spheres. ACS Biomater. Sci. Eng. 2016, 2, 2058-2071. [CrossRef] [PubMed]

25. Feng, J.; Zhang, H.J. Hybrid materials based on lanthanide organic complexes: A review. Chem. Soc. Rev. 2013, 42, 387-410. [CrossRef] 
26. Chen, Y.; Ai, K.L.; Liu, J.H.; Ren, X.Y.; Jiang, C.H.; Lu, L.H. Polydopamine-based coordination nanocomplex for T-1/T-2 dual mode magnetic resonance imaging-guided chemo-photothermal synergistic therapy. Biomaterials 2016, 77, 198-206. [CrossRef]

27. Cai, W.; Gao, H.Y.; Chu, C.C.; Wang, X.Y.; Wang, J.Q.; Zhang, P.F.; Lin, G.; Li, W.G.; Liu, G.; Chen, X.Y. Engineering Phototheranostic Nanoscale Metal-Organic Frameworks for Multimodal Imaging-Guided Cancer Therapy. ACS Appl. Mater. Interfaces 2017, 9 , 2040-2051. [CrossRef]

28. Hyun, H.; Park, M.H.; Owens, E.A.; Wada, H.; Henary, M.; Handgraaf, H.J.M.; Vahrmeijer, A.L.; Frangioni, J.V.; Choi, H.S. Structure-inherent targeting of near-infrared fluorophores for parathyroid and thyroid gland imaging. Nat. Med. 2015, 21, 192-197. [CrossRef]

29. Vahrmeijer, A.L.; Hutteman, M.; van der Vorst, J.R.; van de Velde, C.J.H.; Frangioni, J.V. Image-guided cancer surgery using near-infrared fluorescence. Nat. Rev. Clin. Oncol. 2013, 10, 507-518. [CrossRef]

30. Li, W.W.; Rong, P.F.; Yang, K.; Huang, P.; Sun, K.; Chen, X.Y. Semimetal nanomaterials of antimony as highly efficient agent for photoacoustic imaging and photothermal therapy. Biomaterials 2015, 45, 18-26. [CrossRef]

31. Yang, D.; Yang, G.X.; Yang, P.P.; Lv, R.C.; Gai, S.L.; Li, C.X.; He, F.; Lin, J. Assembly of Au Plasmonic Photothermal Agent and Iron Oxide Nanoparticles on Ultrathin Black Phosphorus for Targeted Photothermal and Photodynamic Cancer Therapy. Adv. Funct. Mater. 2017, 27, 1700371. [CrossRef]

32. Lu, D.; Tao, R.; Wang, Z. Carbon-based materials for photodynamic therapy: A mini-review. Front. Chem. Sci. Eng. 2019, 13, 310-323. [CrossRef]

33. Pinto, A.; Pocard, M. Photodynamic therapy and photothermal therapy for the treatment of peritoneal metastasis: A systematic review. Pleura Peritoneum 2018, 3, doi. [CrossRef] [PubMed]

34. Gupta, N.; Rai, D.B.; Jangid, A.K.; Kulhari, H. A Review of Theranostics Applications and Toxicities of Carbon Nanomaterials. Curr. Drug Metab. 2019, 20, 506-532. [CrossRef] [PubMed]

35. Xu, Y.H.; Shan, Y.L.; Cong, H.L.; Shen, Y.Q.; Yu, B. Advanced Carbon-based Nanoplatforms Combining Drug Delivery and Thermal Therapy for Cancer Treatment. Curr. Pharm. Des. 2018, 24, 4060-4076. [CrossRef] [PubMed]

36. Zhi, D.F.; Yang, T.; O'Hagan, J.; Zhang, S.B.; Donnelly, R.F. Photothermal therapy. J. Control. Release 2020, 325, 52-71. [CrossRef]

37. Wilson, B.C.; Patterson, M.S. The physics, biophysics and technology of photodynamic therapy. Phys. Med. Biol. 2008, 53, R61-R109. [CrossRef]

38. Feng, G.X.; Zhang, G.Q.; Ding, D. Design of superior phototheranostic agents guided by Jablonski diagrams. Chem. Soc. Rev. 2020, 49, 8179-8234. [CrossRef]

39. Ackroyd, R.; Kelty, C.; Brown, N.; Reed, M. The history of photodetection and photodynamic therapy. Photochem. Photobiol. 2001, 74, 656-669. [CrossRef]

40. Triesscheijn, M.; Baas, P.; Schellens, J.H.M.; Stewart, F.A. Photodynamic therapy in oncology. Oncologist 2006, 11, 1034-1044. [CrossRef]

41. Chen, H.Z.; Zhao, Y.L. Applications of Light-Responsive Systems for Cancer Theranostics. Acs Appl. Mater. Interfaces 2018, 10, 21021-21034. [CrossRef]

42. Rozanova, N.; Zhang, J.Z. Photothermal ablation therapy for cancer based on metal nanostructures. Sci. China Ser. B-Chem. 2009, 52, 1559-1575. [CrossRef]

43. Zhang, Y.J.; Zhan, X.L.; Xiong, J.; Peng, S.S.; Huang, W.; Joshi, R.; Cai, Y.; Liu, Y.L.; Li, R.; Yuan, K.; et al. Temperature-dependent cell death patterns induced by functionalized gold nanoparticle photothermal therapy in melanoma cells. Sci. Rep. 2018, 8, 1-9. [CrossRef]

44. Gao, P.; Wang, H.; Cheng, Y. Strategies for efficient photothermal therapy at mild temperatures: Progresses and challenges. Chin. Chem. Lett. 2021, 7, 48-59.

45. Jaffe, H.H.; Miller, A.L. Fates of electronic excitation energy. J. Chem. Educ. 1966, 43, 469. [CrossRef]

46. Dias, L.D.; Mfouo-Tynga, I.S. Learning from Nature: Bioinspired Chlorin-Based Photosensitizers Immobilized on Carbon Materials for Combined Photodynamic and Photothermal Therapy. Biomimetics 2020, 5, 53. [CrossRef] [PubMed]

47. Kushwaha, S.K.S.; Ghoshal, S.; Rai, A.K.; Singh, S. Carbon nanotubes as a novel drug delivery system for anticancer therapy: A review. Braz. J. Pharm. Sci. 2013, 49, 629-643. [CrossRef]

48. Mendes, R.G.; Bachmatiuk, A.; Buchner, B.; Cuniberti, G.; Rummeli, M.H. Carbon nanostructures as multi-functional drug delivery platforms. J. Mater. Chem. B 2013, 1, 401-428. [CrossRef]

49. Maiti, D.; Tong, X.M.; Mou, X.Z.; Yang, K. Carbon-Based Nanomaterials for Biomedical Applications: A Recent Study. Front. Pharmacol. 2019, 9, 1401. [CrossRef]

50. Francisco, W.; Ferreira, F.V.; Ferreira, E.V.; Cividanes, L.D.; Coutinho, A.D.; Thim, G.P. Functionalization of Multi-Walled Carbon Nanotube and Mechanical Property of Epoxy-Based Nanocomposite. J. Aerosp. Technol. Manag. 2015, 7, 289-293. [CrossRef]

51. Dideikin, A.T.; Vul, A.Y. Graphene Oxide and Derivatives: The Place in Graphene Family. Front. Phys. 2019, 6, 105-108. [CrossRef]

52. Georgakilas, V.; Perman, J.A.; Tucek, J.; Zboril, R. Broad Family of Carbon Nanoallotropes: Classification, Chemistry, and Applications of Fullerenes, Carbon Dots, Nanotubes, Graphene, Nanodiamonds, and Combined Superstructures. Chem. Rev. 2015, 115, 4744-4822. [CrossRef] [PubMed]

53. Wang, Z.D.; Hu, T.T.; Liang, R.Z.; Wei, M. Application of Zero-Dimensional Nanomaterials in Biosensing. Front. Chem. 2020, 8, 320. [CrossRef] [PubMed] 
54. Schwerdtfeger, P.; Wirz, L.N.; Avery, J. The topology of fullerenes. Wiley Interdiscip. Rev.-Comput. Mol. Sci. $2015,5,96-145$. [CrossRef] [PubMed]

55. Liu, Z.; Davis, C.; Cai, W.B.; He, L.; Chen, X.Y.; Dai, H.J. Circulation and long-term fate of functionalized, biocompatible single-walled carbon nanotubes in mice probed by Raman spectroscopy. Proc. Natl. Acad. Sci. USA 2008, 105, 1410-1415. [CrossRef]

56. Jasim, D.A.; Menard-Moyon, C.; Begin, D.; Bianco, A.; Kostarelos, K. Tissue distribution and urinary excretion of intravenously administered chemically functionalized graphene oxide sheets. Chem. Sci. 2015, 6, 3952-3964. [CrossRef]

57. Jasim, D.A.; Boutin, H.; Fairclough, M.; Menard-Moyon, C.; Prenant, C.; Bianco, A.; Kostarelos, K. Thickness of functionalized graphene oxide sheets plays critical role in tissue accumulation and urinary excretion: A pilot PET/CT study. Appl. Mater. Today 2016, 4, 24-30. [CrossRef]

58. Gao, C.J.; Dong, P.; Lin, Z.X.; Guo, X.L.; Jiang, B.P.; Ji, S.C.; Liang, H.; Shen, X.C. Near-Infrared Light Responsive Imaging-Guided Photothermal and Photodynamic Synergistic Therapy Nanoplatform Based on Carbon Nanohorns for Efficient Cancer Treatment. Chem.-A Eur. J. 2018, 24, 12827-12837. [CrossRef]

59. Yang, J.X.; Hou, M.F.; Sun, W.S.; Wu, Q.H.; Xu, J.; Xiong, L.Q.; Chai, Y.M.; Liu, Y.X.; Yu, M.H.; Wang, H.L.; et al. Sequential PDT and PTT Using Dual-Modal Single-Walled Carbon Nanohorns Synergistically Promote Systemic Immune Responses against Tumor Metastasis and Relapse. Adv. Sci. 2020, 7, 2001088. [CrossRef]

60. Gao, C.J.; Jian, J.; Lin, Z.X.; Yu, Y.X.; Jiang, B.P.; Chen, H.; Shen, X.C. Hypericin-Loaded Carbon Nanohorn Hybrid for Combined Photodynamic and Photothermal Therapy in Vivo. Langmuir 2019, 35, 8228-8237. [CrossRef]

61. Sun, S.; Chen, J.Q.; Jiang, K.; Tang, Z.D.; Wang, Y.H.; Li, Z.J.; Liu, C.B.; Wu, A.G.; Lin, H.W. Ce6-Modified Carbon Dots for Multimodal-Imaging-Guided and Single-NIR-Laser-Triggered Photothermal/Photodynamic Synergistic Cancer Therapy by Reduced Irradiation Power. ACS Appl. Mater. Interfaces 2019, 11, 5791-5803. [CrossRef] [PubMed]

62. Kalluru, P.; Vankayala, R.; Chiang, C.S.; Hwang, K.C. Nano-graphene oxide-mediated In vivo fluorescence imaging and bimodal photodynamic and photothermal destruction of tumors. Biomaterials 2016, 95, 1-10. [CrossRef] [PubMed]

63. dos Santos, M.S.C.; Gouvea, A.L.; de Moura, L.D.; Paterno, L.G.; de Souza, P.E.N.; Bastos, A.P.; Damasceno, E.A.M.; Veiga-Souza, F.H.; de Azevedo, R.B.; Bao, S.N. Nanographene oxide-methylene blue as phototherapies platform for breast tumor ablation and metastasis prevention in a syngeneic orthotopic murine model. J. Nanobiotechnol. 2018, 16, 1-17. [CrossRef] [PubMed]

64. Ge, J.C.; Jia, Q.Y.; Liu, W.M.; Lan, M.H.; Zhou, B.J.; Guo, L.; Zhou, H.Y.; Zhang, H.Y.; Wang, Y.; Gu, Y.; et al. Carbon Dots with Intrinsic Theranostic Properties for Bioimaging, Red-Light-Triggered Photodynamic/Photothermal Simultaneous Therapy In Vitro and In Vivo. Adv. Healthc. Mater. 2016, 5, 665-675. [CrossRef]

65. Yin, Z.H.; Chen, D.P.; Zou, J.H.; Shao, J.J.; Tang, H.; Xu, H.; Si, W.L.; Dong, X.C. Tumor Microenvironment Responsive OxygenSelf-Generating Nanoplatform for Dual-Imaging Guided Photodynamic and Photothermal Therapy. Chemistryselect 2018, 3, 4366-4373. [CrossRef]

66. Zhang, M.; Wang, W.T.; Zhou, N.L.; Yuan, P.; Su, Y.T.; Shao, M.N.; Chi, C.; Pan, F.Y. Near-infrared light triggered photo-therapy, in combination with chemotherapy using magnetofluorescent carbon quantum dots for effective cancer treating. Carbon 2017, 118, 752-764. [CrossRef]

67. Zhang, M.; Wang, W.T.; Cui, Y.J.; Chu, X.H.; Sun, B.H.; Zhou, N.L.; Shen, J. Magnetofluorescent Fe $\mathrm{O}_{4} /$ carbon quantum dots coated single-walled carbon nanotubes as dual-modal targeted imaging and chemo/photodynamic/photothermal triple-modal therapeutic agents. Chem. Eng. J. 2018, 338, 526-538. [CrossRef]

68. Wang, D.Q.; Zhang, N.; Jing, X.N.; Zhang, Y.; Xu, Y.Z.; Meng, L.J. A tumor-microenvironment fully responsive nano-platform for MRI-guided photodynamic and photothermal synergistic therapy. J. Mater. Chem. B 2020, 8, 8271-8281. [CrossRef] 